\title{
Approximation Results for Urysohn Type Two Dimensional Nonlinear Bernstein Operators
}

\author{
HARUN KARSLI*
}

ABSTRACT. In the present work, our aim of this study is generalization and extension of the theory of interpolation of two dimensional functions to functionals or operators by means of Urysohn type nonlinear operators. In accordance with this purpose, we introduce and study a new type of Urysohn type nonlinear operators. In particular, we investigate the convergence problem for nonlinear operators that approximate the Urysohn type operator in two dimensional case. The starting point of this study is motivated by the important applications that approximation properties of certain families of nonlinear operators have in signal-image reconstruction and in other related fields. We construct our nonlinear operators by using a nonlinear form of the kernels together with the Urysohn type operator values instead of the sampling values of the function.

Keywords: Urysohn integral operators, Nonlinear Bernstein operators, Urysohn type two dimensional nonlinear Bernstein operators.

2010 Mathematics Subject Classification: 41A25, 41A35, 47G10, 47H30.

\section{INTRODUCTION}

For a function defined on the interval $[0,1]$, the Bernstein operators $\left(B_{n} f\right), n \geq 1$, are defined by

$$
\left(B_{n} f\right)(x)=\sum_{k=0}^{n} f\left(\frac{k}{n}\right) p_{n, k}(x), \quad n \geq 1,
$$

where $p_{n, k}(x)=\left(\begin{array}{c}n \\ k\end{array}\right) x^{k}(1-x)^{n-k}$ is the well-known Binomial distribution and called Bernstein basis $(0 \leq x \leq 1)$. These polynomials were introduced by Bernstein [9] in 1912 to give the first constructive proof of the Weierstrass approximation theorem.

For detailed approaches to this operator see the fundamental book of G.G. Lorentz [27].

In his Ph.D. thesis [12] written under the direction of G.G. Lorentz and afterwards in the paper [11] , the famous German mathematician P.L. Butzer considered two dimensional Bernstein polynomials on the square $\square:=\{(x, y): 0 \leq x, y \leq 1\}$ as follows:

$$
B_{n, m}(f ; x, y)=\sum_{k=0}^{n} \sum_{j=0}^{m} f\left(\frac{k}{n}, \frac{j}{m}\right) p_{n, k}(x) p_{m, j}(y)
$$

where $p_{n, k}(t)=\left(\begin{array}{c}n \\ k\end{array}\right) t^{k}(1-t)^{n-k}$.

At the beginning, the theory of approximation is strongly related with the linearity of the operators. But, thanks to the approachs of the Polish mathematician Julian Musielak, see [29],

Received: August 13, 2018; In revised form: August 16, 2018; Accepted: August 19, 2018

*Corresponding author: H. Karsli; karsli_h@ibu.edu.tr

DOI: $10.33205 / \mathrm{cma} .453027$ 
and continuous works of C.Bardaro, G. Vinti and their research group, this theory can be extended to the nonlinear type operators, under some specific assumptions on its kernel functions, see the fundamental book due to Bardaro, Musielak and Vinti [6]. For further reading please see [1]- [5], [13], [14] as well as the monographs [33].

In view of the approaches due to Musielak [29], recently, Karsli-Tiryaki and Altin [23] introduced the following type nonlinear counterpart of the well-known Bernstein operators (1.1);

$$
\left(N B_{n} f\right)(x)=\sum_{k=0}^{n} P_{n, k}\left(x, f\left(\frac{k}{n}\right)\right), 0 \leq x \leq 1, n \in \mathbb{N},
$$

acting on bounded functions $f$ on the interval $[0,1]$, where $P_{n, k}$ satisfy some suitable assumptions. They proved some existence and approximation theorems for the nonlinear Bernstein operators.

Many problems in engineering and mechanics can be transformed into two-dimensional integral equations and corresponding two dimensional integral operators. Especially the integral operators of Fredholm, Volterra, Hammerstein and Urysohn type are used frequently when describing real problems which arise from different sciences, such as physics, engineering, mechanics, theory of elasticity, signal-image reconstruction and in the applications of mathematical physics. So, integral operators of various types form an important and unavoidable part of linear and nonlinear functional analysis.

In 2000, Demkiv [15] and [16] defined and investigated some properties of the following type one and two dimensional Bernstein operators, which are linear with respect to $F$ defined by (2.4);

$$
\left(B_{n} F\right) x(t)=\int_{0}^{1} \sum_{k=0}^{n} f\left(t, s, \frac{k}{n}\right) p_{n, k}(x(s)) d s,
$$

and

$$
\left(B_{n, m} F\right)(x(.), y(.))=\int_{0}^{1} \int_{0}^{1} \sum_{i=0}^{n} \sum_{j=0}^{m} f\left(t, z_{1}, z_{2}, \frac{i}{n}, \frac{j}{m}\right) p_{n, i}\left(x\left(z_{1}\right)\right) p_{n, j}\left(y\left(z_{2}\right)\right) d z_{1} d z_{2} .
$$

In 2017, the author [24] defined the following Urysohn type Meyer-König and Zeller operators;

$$
\begin{aligned}
\left(M_{n} F\right) x(t) & =\int_{0}^{1}\left[\sum_{k=0}^{\infty} f\left(t, s, \frac{k}{k+n}\right) m_{n, k}(x(s))\right] d s \\
\left(M_{n} F\right) 1(t) & =F 1(t)=F(1),
\end{aligned}
$$

where

$$
m_{n, k}(x(s))=\left(\begin{array}{c}
n+k-1 \\
k
\end{array}\right)(x(s))^{k}(1-x(s))^{n},
$$

$n$ is a non-negative integer and $0 \leq x(s)<1$, and obtained some positive results about the convergence problem. 
Very recently in [25] and [26], the author defined and investigated the Urysohn type nonlinear Bernstein operators, having the form

$$
\left(N B_{n} F\right) x(t)=\int_{0}^{1}\left[\sum_{k=0}^{n} P_{k, n}\left(x(s), f\left(t, s, \frac{k}{n}\right)\right)\right] d s, 0 \leq x(s) \leq 1, \quad n \in \mathbb{N} .
$$

The central issue of this paper is to extend the theory of interpolation to functionals and operators by introducing the Urysohn type nonlinear counterpart of the two dimensional Bernstein operators. Afterwards, we investigate the convergence problem for these nonlinear operators.

Due to this importance, in this paper we will deal with integral operators of the two dimensional Urysohn type:

$$
U(x(t), y(t))=\int_{a}^{b} \int_{a}^{b} k(t, s, z, x(s), y(z)) d s d z, \quad t \in[a, b],
$$

where $k$ is a known function and $x$ and $y$ are the unknown functions to be determined.

Let us consider a sequence $N B F=\left(N B_{n} F\right)$ of operators, which we call it Urysohn type nonlinear counterpart of the two dimensional Bernstein operators, having the form:

$$
\begin{aligned}
\left(N B_{n} F\right)(x(t), y(t)) & =\int_{0}^{1} \int_{0}^{1}\left[\sum_{k=0}^{n} \sum_{i=0}^{n} P_{k, i, n}\left(x(s), y(z), f\left(t, s, z, \frac{k}{n}, \frac{i}{n}\right)\right)\right] d s d z, \\
0 & \leq x(s), y(z) \leq 1, \quad n \in \mathbb{N},
\end{aligned}
$$

acting on bounded functions $f$ on $[0,1]^{5}=[0,1] *[0,1] *[0,1] *[0,1] *[0,1]$, where $P_{k, i, n}$ satisfy some suitable assumptions. In particular, we will put $\operatorname{Dom} N B F=\bigcap_{n \in \mathbb{N}} \operatorname{Dom} N B_{n} F$, where $\operatorname{Dom} N B_{n} F$ is the set of all functions $f:[0,1]^{5} \rightarrow \mathbb{R}$ for which the operator is well defined.

\section{PRELIMINARIES AND AUXILIARY RESUlTS}

This section is devoted to collecting some definitions and results which will be needed further on.

Here we consider the following type two dimensional Urysohn integral operator,

$$
F(x(t), y(t))=\int_{0}^{1} \int_{0}^{1} f(t, s, z, x(s), y(z)) d s d z, \quad t \in[0,1]
$$

with unknown kernel $f$ : If such a representation exists, then the kernel function $f(t, s, z, x(),. y()$. is called the two dimensional Green's function, which is strongly related to the functions $x$ and $y$.

Note that in the univariate case, the solution of the following differential equation

$$
D G(x, y)=\delta(x-y),
$$

represents a Green function $G(x, y)$, here $D$ is a differential operator, $\delta$ is the Dirac Delta function and satisfying a boundary condition. Note that

$$
\delta(x)=\frac{d H(x)}{d x},
$$


is true, where

$$
H(x)=\left\{\begin{array}{lll}
1 & , & x \geq 0 \\
0 & , & x<0
\end{array}\right.
$$

is the Heaviside function.

In view of the above relations, we assume that the two dimensional continuous interpolation conditions hold:

$$
F\left(x_{i}(t), y_{j}(t)\right)=\int_{0}^{1} \int_{0}^{1} f\left(t, s, z, x_{i}(s), y_{j}(z)\right) d s d z, \quad t \in[0,1]
$$

where

$$
\begin{aligned}
& x_{i}(s)=\frac{i}{n} H(s-\xi) ; \xi \in[0 ; 1], \\
& y_{j}(z)=\frac{j}{n} H(z-\varsigma) ; \varsigma \in[0 ; 1]
\end{aligned}
$$

and $i, j=0,1,2, \ldots n$.

Taking into account (2.4) and (2.5), by a straightforward calculation the stated identities follow.

$$
\begin{aligned}
F\left(\frac{i}{n} H(s-\xi), \frac{j}{n} H(z-\varsigma)\right) & =\int_{0}^{1} \int_{0}^{1} f\left(t, s, z, \frac{i}{n} H(s-\xi), \frac{j}{n} H(z-\varsigma)\right) d s d z \\
& =\int_{\varsigma}^{1} \int_{\xi}^{1} f\left(t, s, z, \frac{i}{n}, \frac{j}{n}\right) d s d z+\int_{0}^{\varsigma} \int_{\xi}^{1} f\left(t, s, z, \frac{i}{n}, 0\right) d s d z \\
& +\int_{0}^{\varsigma} \int_{0}^{\xi} f(t, s, z, 0,0) d s d z+\int_{\varsigma}^{1} \int_{0}^{\xi} f\left(t, s, z, 0, \frac{j}{n}\right) d s d z
\end{aligned}
$$

and hence

$$
\begin{aligned}
\frac{\partial F\left(\frac{i}{n} H(s-\xi), \frac{j}{n} H(z-\varsigma)\right)}{\partial \varsigma} & =-\int_{\xi}^{1} f\left(t, s, \varsigma, \frac{i}{n}, \frac{j}{n}\right) d s+\int_{\xi}^{1} f\left(t, s, \varsigma, \frac{i}{n}, 0\right) d s \\
& +\int_{0}^{\xi} f(t, s, \varsigma, 0,0) d s-\int_{0}^{\xi} f\left(t, s, \varsigma, 0, \frac{j}{n}\right) d s, \\
\frac{\partial^{2} F\left(\frac{i}{n} H(s-\xi), \frac{j}{n} H(z-\varsigma)\right)}{\partial \xi \partial \varsigma} & =f\left(t, \xi, \varsigma, \frac{i}{n}, \frac{j}{n}\right)-f\left(t, \xi, \varsigma, \frac{i}{n}, 0\right) \\
& +f(t, \xi, \varsigma, 0,0)-f\left(t, \xi, \varsigma, 0, \frac{j}{n}\right) .
\end{aligned}
$$

Say

$$
F_{1}\left(t, \xi, \varsigma, \frac{i}{n}, \frac{j}{n}\right):=\frac{\partial^{2} F\left(\frac{i}{n} H(s-\xi), \frac{j}{n} H(z-\varsigma)\right)}{\partial \xi \partial \varsigma}
$$


According to the above definition together with (2.6) and (2.7), it is possible to construct an approximation operator in order to generalize and extend the theory of interpolation of functions to operators.

In view of (1.2) and (2.4), we introduce the following Urysohn type nonlinear Bernstein operators;

$$
\left(N B_{n} F\right)(x(t), y(t))=\int_{0}^{1} \int_{0}^{1}\left[\sum_{k=0}^{n} \sum_{i=0}^{n} P_{k, i, n}\left(x(s), y(z), f\left(t, s, z, \frac{k}{n}, \frac{i}{n}\right)\right)\right] d s d z
$$

where $n$ is a non-negative integer, $P_{k, i, n}$ satisfy some suitable assumptions.and $0 \leq x(s), y(z) \leq$ 1 .

Now, we assemble the main definitions and notations which will be used throughout the paper.

Let $X$ be the set of all bounded Lebesgue measurable functions $f:[0,1]^{5} \rightarrow \mathbb{R}_{0}^{+}=[0, \infty)$.

Let $\Psi$ be the class of all functions $\psi: \mathbb{R}_{0}^{+} \rightarrow \mathbb{R}_{0}^{+}$such that the function $\psi$ is continuous and concave with $\psi(0)=0, \psi(u)>0$ for $u>0$.

We now introduce a sequence of functions. Let $\left\{P_{k, i, n}\right\}_{n \in \mathbb{N}}$ be a sequence of functions $P_{k, i, n}$ : $[0,1] \times[0,1] \times \mathbb{R} \rightarrow \mathbb{R}$ defined by

$$
P_{k, i, n}(t, l, u)=p_{k, n}(t) p_{i, n}(l) H_{n}(u)
$$

for every $t, l \in[0,1], u \in \mathbb{R}$, where $H_{n}: \mathbb{R} \rightarrow \mathbb{R}$ is such that $H_{n}(0)=0$ and $p_{k, n}(\bullet)$ is the Bernstein basis. For simplicity we will write

$$
P_{k, i, n}(t, l):=p_{k, n}(t) p_{i, n}(l) .
$$

In what follows, throughout the paper, we assume that $\mu: \mathbb{N} \rightarrow \mathbb{R}^{+}$is an increasing and continuous function such that $\lim _{n \rightarrow \infty} \mu(n)=\infty$.

First of all we assume that the following conditions hold:

a) $H_{n}: \mathbb{R} \rightarrow \mathbb{R}$ is such that

$$
\left|H_{n}(u)-H_{n}(v)\right| \leq \psi(|u-v|),
$$

holds for every $u, v \in \mathbb{R}$, for every $n \in \mathbb{N}$. That is, $H_{n}$ satisfies a $(L-\Psi)$ Lipschitz condition.

$b$ ) Denoting by $r_{n}(u):=H_{n}(u)-u, u \in \mathbb{R}$ and $n \in \mathbb{N}$, such that for $n$ sufficiently large

$$
\sup _{u}\left|r_{n}(u)\right|=\sup _{u}\left|H_{n}(u)-u\right| \leq \frac{1}{\mu(n)},
$$

holds.

Following our announced aim, in this part we recall results regarding the univariate and linear case of the celerated Bernstein polynomials.

Lemma 2.1. For $\left(B_{n} t^{s}\right)(x, y), s=0,1,2$, one has

$$
\begin{aligned}
\left(B_{n} 1\right)(x, y) & =1 \\
\left(B_{n} t\right)(x, y) & =x \\
\left(B_{n} t^{2}\right)(x, y) & =x^{2}+\frac{x(1-x)}{n} .
\end{aligned}
$$


For proof of this Lemma see [27].

By direct calculation, we find the following equalities:

$$
\left(B_{n}(t-x)^{2}\right)(x, y)=\frac{x(1-x)}{n},\left(B_{n}(t-x)\right)(x, y)=0 .
$$

Lemma 2.2. For the central moments of order $m \in N_{0}$

$$
T_{n, m}(x):=\sum_{k=0}^{n}(k-n x)^{m} p_{k, n}(x),
$$

for each $m=0,1, \ldots$ there is a constant $A_{m}$ such that

$$
0 \leq T_{n, 2 m}(x) \leq A_{m} n^{m} .
$$

The presented well-known inequality can be found in [17].

\section{CONVERGENCE PROPERTY}

We now introduce some notations and structural hypotheses, which will be fundamental in proving our convergence theorems.

Let $C[0,1]$ the Banach space of continuous functions $u:[0,1] \rightarrow R$ endowed with the norm

$$
\|u\|=\sup \{|u(x)|: x \in[0,1]\} .
$$

Definition 3.1. Let $f \in C\left([a, b]^{5}\right)$ and $\delta>0$ be given. Then the complete modulus of continuity is given by;

$$
\omega(\delta)=\sup _{\sqrt{\left(u_{1}-u_{2}\right)^{2}+\left(v_{1}-v_{2}\right)^{2}} \leq \delta}\left|f\left(t, s, z, u_{1}, v_{1}\right)-f\left(t, s, z, u_{2}, v_{2}\right)\right| .
$$

Further on, the first and second partial modulus of continuity are given by

$$
\begin{gathered}
\omega_{1}\left(\delta_{1}, 0\right)=\sup _{\left|u_{1}-u_{2}\right| \leq \delta_{1}}\left|f\left(t, s, z, u_{1}, v_{1}\right)-f\left(t, s, z, u_{2}, v_{1}\right)\right|, \\
\omega_{2}(0, \eta)=\sup _{\left|v_{1}-v_{2}\right| \leq \eta}\left|f\left(t, s, z, u_{1}, v_{1}\right)-f\left(t, s, z, u_{1}, v_{2}\right)\right| .
\end{gathered}
$$

Recall that $\omega(f ; \delta)$ has the following properties;

(i) Let $\lambda \in \mathbb{R}^{+}$, then $\omega(f ; \lambda \delta) \leq(\lambda+1) \omega(f ; \delta)$,

(ii) $\lim _{\delta \rightarrow 0^{+}} \omega(f ; \delta)=0$,

(iii) $\left|f\left(t, s, z, u_{1}, v_{1}\right)-f\left(t, s, z, u_{2}, v_{2}\right)\right| \leq \omega(\delta)\left(1+\frac{\sqrt{\left(u_{1}-u_{2}\right)^{2}+\left(v_{1}-v_{2}\right)^{2}}}{\delta}\right)$,

Note that the same properties also hold for partial moduli of continuity.

We are now ready to establish one of the main results of this study:

Theorem 3.1. Let $F$ be the Urysohn integral operator with $0 \leq x(s), y(z) \leq 1$. Then $\left(N B_{n} F\right)$ converges to $F$ uniformly in $x, y \in C[0,1]$. That is

$$
\lim _{n \rightarrow \infty}\left\|\left(N B_{n} F\right)(x(t), y(t))-F(x(t), y(t))\right\|_{C\left([0,1]^{2}\right)}=0 .
$$


Proof. In view of the definition of the operator (2.8), by considering (2.4), (2.9), (2.6) and (2.7), we have

$$
\begin{aligned}
& \left|\left(N B_{n} F\right)(x(t), y(t))-F(x(t), y(t))\right| \\
= & \left|\int_{0}^{1} \int_{0}^{1}\left[\sum_{k=0}^{n} \sum_{i=0}^{n} P_{k, i, n}\left(x(s), y(z), f\left(t, s, z, \frac{k}{n}, \frac{i}{n}\right)\right)\right] d s d z-F(x(t), y(t))\right| \\
\leq & \int_{0}^{1} \int_{0}^{1} \sum_{k=0}^{n} \sum_{i=0}^{n} P_{k, i, n}(x, y)\left|H_{n}\left(f\left(t, s, z, \frac{k}{n}, \frac{i}{n}\right)\right)-H_{n}(f(t, s, z, x(s), y(z)))\right| d s d z \\
+ & \int_{0}^{1} \int_{0}^{1} \sum_{k=0}^{n} P_{k, i, n}(x, y)\left|H_{n}(f(t, s, z, x(s), y(z)))-f(t, s, z, x(s), y(z))\right| d s d z \\
:= & I_{1}+I_{2} .
\end{aligned}
$$

By assumption $b$ ) $I_{2}$ tends to zero as $n \rightarrow \infty$. In fact

$$
\begin{aligned}
I_{2} & =\int_{0}^{1} \int_{0}^{1} \sum_{k=0}^{n} \sum_{i=0}^{n} P_{k, i, n}(x, y)\left|H_{n}(f(t, s, z, x(s), y(z)))-f(t, s, z, x(s), y(z))\right| d s d z \\
& \leq \int_{0}^{1} \int_{0}^{1} \sum_{k=0}^{n} \sum_{i=0}^{n} P_{k, i, n}(x, y) \frac{1}{\mu(n)} d s d z \\
& =\frac{1}{\mu(n)},
\end{aligned}
$$

which tends to zero as $n \rightarrow \infty$. Now, it is sufficient to evaluate the term $I_{1}$. Using the definition of the function $F_{1}(t, s, z, x(s), y(z))$, by concavity of the function $\psi$, and using Jensen inequality, we obtain

$$
\begin{aligned}
I_{1} & \leq \int_{0}^{1} \int_{0}^{1} \sum_{k=0}^{n} \sum_{i=0}^{n} P_{k, i, n}(x, y) \psi\left(\left|f\left(t, s, z, \frac{k}{n}, \frac{i}{n}\right)-f(t, s, z, x(s), y(z))\right|\right) d s d z \\
& \leq \psi\left(\int_{0}^{1} \int_{0}^{1} \sum_{k=0}^{n} \sum_{i=0}^{n} P_{k, i, n}(x, y)\left|f\left(t, s, z, \frac{k}{n}, \frac{i}{n}\right)-f(t, s, z, x(s), y(z))\right| d s d z\right)
\end{aligned}
$$




$$
\begin{aligned}
& \leq \psi\left\{\int_{0}^{1} \int_{0}^{1} \sum_{k=0}^{n} \sum_{i=0}^{n} P_{k, i, n}(x, y)\left|F_{1}(t, s, z, x(s), y(z))-F_{1}\left(t, s, z, \frac{k}{n}, \frac{i}{n}\right)\right| d s d z\right. \\
& +\int_{0}^{1} \int_{0}^{1}\left|f(t, s, z, x(s), 0)-\sum_{k=0}^{n} p_{k, n}(x(s)) f\left(t, s, z, \frac{k}{n}, 0\right)\right| d s d z \\
& \left.+\int_{0}^{1} \int_{0}^{1}\left|f(t, s, z, 0, y(z))-\sum_{i=0}^{n} p_{i, n}(y(z)) f\left(t, s, z, 0, \frac{i}{n}\right)\right| d s d z\right\} \\
& : \leq I_{1,1}+I_{1,2}+I_{1,3} .
\end{aligned}
$$

Let us divide the first term into four parts as;

$$
\begin{aligned}
I_{1,1} & =\psi\left(\int_{0}^{1} \int_{0}^{1} \sum_{k=0}^{n} \sum_{i=0}^{n} P_{k, i, n}(x, y)\left|F_{1}(t, s, z, x(s), y(z))-F_{1}\left(t, s, z, \frac{k}{n}, \frac{i}{n}\right)\right| d s d z\right) \\
& : \leq I_{1,1,1}+I_{1,1,2}+I_{1,1,3}+I_{1,1,4},
\end{aligned}
$$

where

$I_{1,1,1}$

$$
=\psi\left(\int_{0}^{1} \int_{0}^{1} \sum_{\left|\frac{k}{n}-x(s)\right|<\delta_{1}} \sum_{\left|\frac{i}{n}-y(z)\right|<\delta_{2}} P_{k, i, n}(x, y)\left|F_{1}(t, s, z, x(s), y(z))-F_{1}\left(t, s, z, \frac{k}{n}, \frac{i}{n}\right)\right| d s d z\right),
$$$$
I_{1,1,2}
$$$$
=\psi\left(\int_{0}^{1} \int_{0}^{1} \sum_{\left|\frac{k}{n}-x(s)\right|<\delta_{1}\left|\frac{i}{n}-y(z)\right| \geq \delta_{2}} \sum_{k, i, n}(x, y)\left|F_{1}(t, s, z, x(s), y(z))-F_{1}\left(t, s, z, \frac{k}{n}, \frac{i}{n}\right)\right| d s d z\right),
$$$$
I_{1,1,3}
$$$$
=\psi\left(\int_{0}^{1} \int_{0}^{1} \sum_{\left|\frac{k}{n}-x(s)\right| \geq \delta_{1}\left|\frac{i}{n}-y(z)\right|<\delta_{2}} \sum_{k, i, n}(x, y)\left|F_{1}(t, s, z, x(s), y(z))-F_{1}\left(t, s, z, \frac{k}{n}, \frac{i}{n}\right)\right| d s d z\right),
$$

and

$$
\begin{aligned}
I_{1,1,4} & \left(\int_{0}^{1} \int_{0}^{1} \sum_{\left|\frac{k}{n}-x(s)\right| \geq \delta_{1}} \sum_{\left|\frac{i}{n}-y(z)\right| \geq \delta_{2}} P_{k, i, n}(x, y)\left|F_{1}(t, s, z, x(s), y(z))-F_{1}\left(t, s, z, \frac{k}{n}, \frac{i}{n}\right)\right| d s d z\right) .
\end{aligned}
$$


Since $x, y \in C[0,1]$, then there exist $\delta_{1}, \delta_{2}>0$ such that

$$
\left|F_{1}(t, s, z, x(s), y(z))-F_{1}\left(t, s, z, \frac{k}{n}, \frac{i}{n}\right)\right|<\epsilon
$$

holds true when $\left|\frac{k}{n}-x(s)\right|<\delta_{1}$ and $\left|\frac{i}{n}-y(z)\right|<\delta_{2}$. So one can easily obtain

$$
I_{1,1,1}<\psi(\epsilon) \text {. }
$$

As to the other terms

$$
\left|F_{1}(t, s, z, x(s), y(z))-F_{1}\left(t, s, z, \frac{k}{n}, \frac{i}{n}\right)\right| \leq 2 M
$$

holds true for some $M>0$, when $\left|\frac{k}{n}-x(s)\right| \geq \delta_{1}$ or $\left|\frac{i}{n}-y(z)\right| \geq \delta_{2}$.

In view of Lemma 2 , we obtain

$$
\begin{aligned}
I_{1,1,2} & =\psi\left(\int_{0}^{1} \int_{0}^{1} \sum_{\left|\frac{k}{n}-x(s)\right|<\delta_{1}} \sum_{\left|\frac{i}{n}-y(z)\right| \geq \delta_{2}} P_{k, i, n}(x, y)\left|F_{1}(t, s, z, x(s), y(z))-F_{1}\left(t, s, z, \frac{k}{n}, \frac{i}{n}\right)\right| d s d z\right) \\
& \leq \psi\left(2 M \int_{0}^{1} \int_{0}^{1} \sum_{\left|\frac{k}{n}-x(s)\right|<\delta_{1}} \sum_{\left|\frac{i}{n}-y(z)\right| \geq \delta_{2}}\left(\frac{i-n y(z)}{\delta_{2}}\right)^{2} P_{k, i, n}(x, y) d s d z\right) \\
& \leq \psi\left(2 M \int_{0}^{1} \int_{0}^{1} \sum_{\left|\frac{k}{n}-x(s)\right|<\delta_{1}}\left(\frac{i-n y(z)}{\delta_{2}}\right)^{2} P_{k, i, n}(x, y) d s d z\right) \\
& \leq \psi\left(\frac{2 M}{\delta_{2}^{2}} \frac{A_{1}}{n}\right) .
\end{aligned}
$$

Similarly one has

$$
I_{1,1,3} \leq \psi\left(\frac{2 M}{\delta^{2}} \frac{A_{1}}{n}\right)
$$

and

$$
I_{1,1,4} \leq \psi\left(\frac{2 M}{\delta_{1}^{2} \delta_{2}^{2}} \frac{A_{1}^{2}}{n^{2}}\right) .
$$

Collecting these estimates we have

$\left|\left(N B_{n} F\right)(x(t), y(t))-F(x(t), y(t))\right| \leq \psi(\epsilon)+\psi\left(\frac{2 M A_{1}}{n \delta_{1}^{2}}\right)+\psi\left(\frac{2 M A_{1}}{n \delta_{2}^{2}}\right)+\psi\left(\frac{2 M}{\delta_{1}^{2} \delta_{2}^{2}} \frac{A_{1}^{2}}{n^{2}}\right)+\frac{1}{\mu(n)}$.

That is

$$
\lim _{n \rightarrow \infty}\left\|\left(N B_{n} F\right)(x(t), y(t))-F(x(t), y(t))\right\|_{C\left([0,1]^{2}\right)}=0 .
$$

This completes the proof.

Theorem 3.2. Let $F$ be the Urysohn integral operator with $x, y \in C[0,1]$, and $0 \leq x(s), y(z) \leq 1$. Then

$$
\left|\left(N B_{n} F\right)(x(t), y(t))-F(x(t), y(t))\right| \leq 2 \psi(\omega(f ; \delta))+\frac{1}{\mu(n)}
$$

holds true, where $\delta=\sqrt{\frac{2 A_{1}}{n}}$. 
Proof. Clearly one has

$$
\begin{aligned}
& \left|\left(N B_{n} F\right)(x(t), y(t))-F(x(t), y(t))\right| \\
\leq & \int_{0}^{1} \int_{0}^{1} \sum_{k=0}^{n} \sum_{i=0}^{n} P_{k, i, n}(x, y)\left|H_{n}\left(f\left(t, s, z, \frac{k}{n}, \frac{i}{n}\right)\right)-H_{n}(f(t, s, z, x(s), y(z)))\right| d s d z \\
& +\frac{1}{\mu(n)} \\
\text { (3.11) : } \quad= & I_{n, 1}(x)+\frac{1}{\mu(n)},
\end{aligned}
$$

say. Since $x, y \in C[0,1]$ we can re-write (3.11) as follows

$$
\begin{aligned}
& I_{n, 1}(x) \leq \int_{0}^{1} \int_{0}^{1} \sum_{k=0}^{n} \sum_{i=0}^{n} P_{k, i, n}(x, y) \psi\left(\left|f\left(t, s, z, \frac{k}{n}, \frac{i}{n}\right)-f(t, s, z, x(s), y(z))\right|\right) d s d z \\
& \leq \int_{0}^{1} \int_{0}^{1} \sum_{k=0}^{n} \sum_{i=0}^{n} P_{k, i, n}(x, y) \psi(\omega(f ; \delta)) d s d z \\
& \leq \psi\left(\int_{0}^{1} \int_{0}^{1} \sum_{k=0}^{n} \sum_{i=0}^{n} P_{k, i, n}(x, y) \omega(f ; \delta) d s d z\right) \\
& \leq \psi\left(\int_{0}^{1} \int_{0}^{1} \sum_{k=0}^{n} \sum_{i=0}^{n} P_{k, i, n}(x, y)\left(\frac{\sqrt{\left(\frac{k}{n}-x(s)\right)^{2}+\left(\frac{i}{n}-y(z)\right)^{2}}}{\delta}+1\right) \omega(f ; \delta) d s d z\right) \\
& =\psi\left(\omega(f ; \delta) \int_{0}^{1} \int_{0}^{1} \sum_{k=0}^{n} \sum_{i=0}^{n} P_{k, i, n}(x, y) \frac{\sqrt{\left(\frac{k}{n}-x(s)\right)^{2}+\left(\frac{i}{n}-y(z)\right)^{2}}}{\delta} d s d z\right) \\
& +\psi\left(\omega(f ; \delta) \int_{0}^{1} \int_{0}^{1} \sum_{k=0}^{n} \sum_{i=0}^{n} P_{k, i, n}(x, y) d s d z\right) \\
& \leq \psi\left(\frac{\omega(f ; \delta)}{\delta} \int_{0}^{1} \int_{0}^{1}\left(\sum_{k=0}^{n} \sum_{i=0}^{n} P_{k, i, n}(x, y)\left[\left(\frac{k}{n}-x(s)\right)^{2}+\left(\frac{i}{n}-y(z)\right)^{2}\right]\right)^{1 / 2} d s d z\right) \\
& +\psi(\omega(f ; \delta)) \\
& \leq \psi\left(\frac{\omega(f ; \delta)}{\delta}\left[\frac{2 A_{1}}{n}\right]^{1 / 2}\right)+\psi(\omega(f ; \delta)) .
\end{aligned}
$$

Taking into account that $\omega(f ; \delta)$ is the modulus of continuity defined as (3.10). If we choose

$$
\delta=\sqrt{\frac{2 A_{1}}{n}},
$$

then one can obtain the desired estimate, namely,

$$
\left|\left(N B_{n} F\right)(x(t), y(t))-F(x(t), y(t))\right| \leq 2 \psi(\omega(f ; \delta))+\frac{1}{\mu(n)} .
$$

Thus the proof is now complete. 
Theorem 3.3. Let $F$ be the Urysohn integral operator with $x, y \in C[0,1]$, and $0 \leq x(s), y(z) \leq 1$. Then

$$
\begin{aligned}
\left|\left(N B_{n} F\right)(x(t), y(t))-F(x(t), y(t))\right| & \leq 2\left[\psi\left(\omega_{1}\left(f ;\left[\frac{A_{1}}{n}\right]^{1 / 2}\right)\right)+\psi\left(\omega_{2}\left(f ;\left[\frac{A_{1}}{n}\right]^{1 / 2}\right)\right)\right] \\
& +\frac{1}{\mu(n)}
\end{aligned}
$$

holds true.

Proof. Clearly one has

$$
\begin{aligned}
& \left|\left(N B_{n} F\right)(x(t), y(t))-F(x(t), y(t))\right| \\
& \leq \int_{0}^{1} \int_{0}^{1} \sum_{k=0}^{n} \sum_{i=0}^{n} P_{k, i, n}(x, y)\left|H_{n}\left(f\left(t, s, z, \frac{k}{n}, \frac{i}{n}\right)\right)-H_{n}(f(t, s, z, x(s), y(z)))\right| d s d z+\frac{1}{\mu(n)} \\
& =\int_{0}^{1} \int_{0}^{1} \sum_{k=0}^{n} \sum_{i=0}^{n} P_{k, i, n}(x, y)\left|\begin{array}{c}
H_{n}\left(f\left(t, s, z, \frac{k}{n}, \frac{i}{n}\right)\right)-H_{n}\left(f\left(t, s, z, x(s), \frac{i}{n}\right)\right) \\
+H_{n}\left(f\left(t, s, z, x(s), \frac{i}{n}\right)\right)-H_{n}(f(t, s, z, x(s), y(z)))
\end{array}\right| d s d z+\frac{1}{\mu(n)} \\
& \leq \int_{0}^{1} \int_{0}^{1} \sum_{k=0}^{n} \sum_{i=0}^{n} P_{k, i, n}(x, y)\left|H_{n}\left(f\left(t, s, z, \frac{k}{n}, \frac{i}{n}\right)\right)-H_{n}\left(f\left(t, s, z, x(s), \frac{i}{n}\right)\right)\right| d s d z \\
& +\int_{0}^{1} \int_{0}^{1} \sum_{k=0}^{n} \sum_{i=0}^{n} P_{k, i, n}(x, y)\left|H_{n}\left(f\left(t, s, z, x(s), \frac{i}{n}\right)\right)-H_{n}(f(t, s, z, x(s), y(z)))\right| d s d z \\
& +\frac{1}{\mu(n)} \\
& :=I_{n, 1}(x)+I_{n, 2}(x)+\frac{1}{\mu(n)},
\end{aligned}
$$

say. Since $x, y \in C[0,1]$ we can re-write (3.11) as follows: By concavity of the function $\psi$, and using Jensen inequality, we obtain

$$
\begin{aligned}
I_{n, 1}(x) & =\int_{0}^{1} \int_{0}^{1} \sum_{k=0}^{n} \sum_{i=0}^{n} P_{k, i, n}(x, y)\left|H_{n}\left(f\left(t, s, z, \frac{k}{n}, \frac{i}{n}\right)\right)-H_{n}\left(f\left(t, s, z, x(s), \frac{i}{n}\right)\right)\right| d s d z \\
& \leq \int_{0}^{1} \int_{0}^{1} \sum_{k=0}^{n} \sum_{i=0}^{n} P_{k, i, n}(x, y) \psi\left(\omega_{1}\left(f ;\left|\frac{k}{n}-x(s)\right|\right)\right) d s d z \\
& \leq \psi\left(\int_{0}^{1} \int_{0}^{1} \sum_{k=0}^{n} \sum_{i=0}^{n} P_{k, i, n}(x, y) \omega_{1}\left(f ;\left|\frac{k}{n}-x(s)\right|\right) d s d z\right)
\end{aligned}
$$


Since $\psi$ is non decreasing, then one has

$$
\begin{aligned}
I_{n, 1}(x) & \leq \psi\left(\int_{0}^{1} \int_{0}^{1} \sum_{k=0}^{n} \sum_{i=0}^{n} P_{k, i, n}(x, y)\left(\frac{\sqrt{\left(\frac{k}{n}-x(s)\right)^{2}}}{\delta_{1}}+1\right) \omega_{1}\left(f ; \delta_{1}\right) d s d z\right) \\
& \leq \psi\left(\frac{\omega_{1}\left(f ; \delta_{1}\right)}{\delta_{1}}\left[\frac{A_{1}}{n}\right]^{1 / 2}\right)+\psi\left(\omega_{1}\left(f ; \delta_{1}\right)\right) .
\end{aligned}
$$

Similarly

$$
I_{n, 1}(x) \leq \psi\left(\frac{\omega_{2}(f ; \eta)}{\eta}\left[\frac{A_{1}}{n}\right]^{1 / 2}\right)+\psi\left(\omega_{2}(f ; \eta)\right) .
$$

If we choose $\delta=\eta=\left[\frac{A_{1}}{n}\right]^{1 / 2}$, so we get the desired estimate.

\section{REFERENCES}

[1] C. Bardaro, I. Mantellini, A Voronovskaya-type theorem for a general class of discrete operators, Rocky Mountain J. Math. 39 (5) (2009) 1411-1442.

[2] C. Bardaro, I. Mantellini, Pointwise convergence theorems for nonlinear Mellin convolution operators. Int. J. Pure Appl. Math. 27 (2006), no. 4, 431-447.

[3] C. Bardaro, I. Mantellini, On the reconstruction of functions by means of nonlinear discrete operators. J. Concr. Appl. Math. 1 (2003), no. 4, 273-285.

[4] C. Bardaro, I. Mantellini, Approximation properties in abstract modular spaces for a class of general samplingtype operators. Appl. Anal. 85 (2006), no. 4, 383-413.

[5] C. Bardaro, G. Vinti, Urysohn integral operators with homogeneous kernel: approximation properties in modular spaces. Comment. Math. (Prace Mat.) 42 (2002), no. 2, 145-182.

[6] C. Bardaro, J. Musielak, G. Vinti, Nonlinear Integral Operators and Applications, De Gruyter Series in Nonlinear Analysis and Applications, Vol. 9, xii + 201 pp., 2003.

[7] C. Bardaro, H. Karsli and G. Vinti, Nonlinear integral operators with homogeneous kernels: pointwise approximation theorems, Applicable Analysis, Vol. 90, Nos. 3-4, March-April (2011), 463-474.

[8] C. Bardaro, H. Karsli and G. Vinti, On pointwise convergence of linear integral operators with homogeneous kernels , Integral Transforms and Special Functions, 19(6), (2008), 429-439.

[9] S. N Bernstein, Demonstration du Thĕoreme de Weierstrass fondĕe sur le calcul des probabilitĕs, Comm. Soc. Math. Kharkow 13, (1912/13), 1-2.

[10] R. Bojanic and F. Cheng, Rate of convergence of Bernstein polynomials for functions with derivatives of bounded variation, J. Math. Anal.and Appl., 141, (1989), 136-151.

[11] P. L. Butzer, On two dimensional Bernstein polynomials, Canad. J. Math. 5 (1953) 107-113.

[12] P. L. Butzer, On Bernstein Polynomials, Ph.D. Thesis, University of Toronto in November, (1951).

[13] D. Costarelli, G. Vinti, Degree of approximation for nonlinear multivariate sampling Kantorovich operators on some functions spaces. Numer. Funct. Anal. Optim. 36 (2015), no. 8, 964-990.

[14] D. Costarelli, G. Vinti, Approximation by nonlinear multivariate sampling Kantorovich type operators and applications to image processing. Numer. Funct. Anal. Optim. 34 (2013), no. 8, 819-844.

[15] I. Demkiv, On approximation of Urison operator with operator polynomials of Bernstein type / / Visn. Lviv univ. Ser. appl. math. and inform, (2000), Vol. 2. p.26-30.

[16] I. Demkiv, On approximation of Urison operator with operator polynomials of Bernstein type in the case two variables / Visn. Lviv polytechnic National University. Ser. appl. math. (2000), p.111-115.

[17] R.A. DeVore, G.G. Lorentz, Constructive Approximation, Springer, New York, 1993.

[18] H. Karsli, Some convergence results for nonlinear singular integral operators, Demonstratio. Math., Vol. XLVI No 4, 729-740 (2013).

[19] H. Karsli, Convergence and rate of convergence by nonlinear singular integral operators depending on two parameters, Appl. Anal. 85(6,7), (2006), 781-791.

[20] H. Karsli, I.U. Tiryaki and H.E. Altin, On convergence of certain nonlinear Bernstein operators. Filomat 30 (2016), no. 1, 141-155.

[21] H. Karsli, H.E. Altin, A Voronovskaya-type theorem for a certain nonlinear Bernstein operators. Stud. Univ. BabeşBolyai Math. 60 (2015), no. 2, 249-258. 
[22] H. Karsli, H.E. Altin, Convergence of certain nonlinear counterpart of the Bernstein operators. Commun. Fac. Sci. Univ. Ank. Sér. A1 Math. Stat. 64 (2015), no. 1, 75-86.

[23] H. Karsli, I.U. Tiryaki and H.E. Altin, Some approximation properties of a certain nonlinear Bernstein operators, Filomat 28:6 (2014), 1295-1305.

[24] H. Karsli, Approximation by Urysohn type Meyer-König and Zeller operators to Urysohn integral operators. Results Math. 72 (2017), no. 3, 1571-1583.

[25] H. Karsli, Approximation results for Urysohn type nonlinear Bernstein operators, Advances in Summability and Approximation Theory, Book Chapter, Springer-Verlag, (2018), accepted.

[26] H. Karsli, Voronovskaya-type theorems for Urysohn type nonlinear Bernstein operators, Mathematical Methods in the Applied Sciences, (2018), accepted.

[27] G.G. Lorentz, Bernstein Polynomials, University of Toronto Press,Toronto (1953).

[28] V.L. Makarov and V.V. Khlobystov, On the identification of Nonlinear operators and its application, BEM IX, (1987), No. 1, 43 - 58.

[29] J. Musielak, On some approximation problems in modular spaces, In Constructive Function Theory 1981, (Proc. Int. Conf., Varna, June 1-5, 1981), pp. 455-461, Publ. House Bulgarian Acad. Sci., Sofia (1983).

[30] P.S. Urysohn, Sur une classe d'equations integrales non lineaires, Mat. Sb., (1923), 31, 236 - 255.

[31] P.S. Urysohn, On a type of nonlinear integral equation. Mat. Sb.,(1924), 31, 236-355

[32] A.M. Wazwaz, Linear and Nonlinear Integral Equations: Methods and Applications, Beijing and Springer - Verlag Berlin, (2011), Higher Education press.

[33] P. P. Zabreiko, A.I. Koshelev, M.A. Krasnosel'skii, S.G. Mikhlin, L.S. Rakovscik and V.Ja. Stetsenko, Integral Equations: A Reference Text, Noordhoff Int. Publ., Leyden, (1975).

BOLU ABANT IZZET BAYSAL UNIVERSITY

FACULTY OF SCIENCE AND ARTS

DEPARTMENT OF MATHEMATICS

14280, GÖLKÖY-BOLU, TURKEY

E-mail address: karsli_h@ibu.edu.tr 